\title{
SATISFAÇÃO DE RESIDENTES COM SEU LUGAR: DEFINIÇÃO E PROPOSTA DE UMA ESCALA DE MENSURAÇÃO DE MÚLTIPLOS ITENS
}

RESIDENTS' SATISFACTION WITH THE PLACE WHERE THEY LIVE: DEFINITION AND PROPOSAL OF A MEASURING SCALE OF MULTIPLE ITEMS

LA SATISFACCIÓN DE LOS RESIDENTES CON SU LUGAR: DEFINICIÓN Y PROPUESTA DE UNA ESCALA DE MEDICIÓN DE MÚLTIPLES ÍTEMS

Fabiana Gama de Medeiros

Universidade Federal da Paraíba

gmfabiana@hotmail.com

(Professora na UFPB, Me. Administração, Esp. Consultoria Organizacional, Grad.

Administração e Turismo)

Francisco José da Costa

Universidade Federal da Paraíba

franzecosta@gmail.com

(Professor na UFPB, Dr. Administração, Me. Administração, Grad. Administração e Estatística)

Data de Submissão: 03/10/2015

Data de Aprovação:06/11/2015

RESUMO: Este estudo analisa o construto 'satisfação dos residentes com o lugar', com a finalidade específica de propor uma escala para sua mensuração. O pressuposto foi de que a atratividade de um lugar é evidenciada, em grande parte, pela satisfação que seus residentes têm, de modo que se faz necessário monitorar e empreender esforços para elevar o nível de satisfação deste grupo de stakeholders. A partir de uma revisão da literatura da área, definiu-se o construto e avaliaram-se os principais indicadores de satisfação. Em seguida, foi empreendido um esforço de construção de uma métrica, seguindo os passos convencionais de construção de escalas para construtos com mensuração refletiva. Para validação empírica, foram coletados dados das cidades do estado da Paraíba, Brasil: João Pessoa, Campina Grande, Guarabira, e algumas outras pequenas cidades 
circunvizinhas. Inicialmente foi feita uma primeira atividade de amostragem com a finalidade de limpeza da escala, e a segunda amostragem foi realizada no sentido de validar a escala. A escala final proposta é unidimensional de quatro itens e pode ser utilizada não apenas para cidades, como também para bairros e/ou regiões. A proposição dessa escala tem direta implicação prática na atuação dos gestores de lugares no sentido de oferecer um instrumento de mensuração da satisfação de seus residentes. Academicamente, preenche uma lacuna na literatura da área em termos de métrica na área de satisfação de lugares.

Palavras-chave: Residentes. Satisfação. Mensuração.

ABSTRACT: This study examines the construct 'resident's satisfaction with the place where they live', with the specific aim of proposing a scale for measuring this satisfaction. It is based on the premise that the attractiveness of a place is largely evidenced in the residents' satisfaction with the place where they live, thus, it is necessary to monitor and make efforts to raise the level of satisfaction of this group of stakeholders. Based on a literature review, we defined the construct and evaluated the key indicators of satisfaction. We then constructed a metric, following the conventional steps for constructing scales for the development of reflective constructs. For the empirical validation, data were collected from the towns of João Pessoa, Campina Grande, Guarabira, and other small, neighboring towns, in the state of Paraíba, Brazil. An initial sampling was conducted to minimize outliers in the scale, and a second sampling was conducted to validate it. The final proposed scale is a four-item single-dimensional scale that can be applied not only to towns and cities, but also to neighborhoods and/or regions. The proposed scale has direct practical implications for the practices of town manager, as it offers an instrument for measuring residents' satisfaction. From and academic point of view, this study fills in a gap in the literature in the area, providing a measurement scale in the area of satisfaction with places.

Keywords: Residents. Satisfaction. Measurement.

RESUMEN: Este estudio analiza el constructo 'satisfacción de los residentes con el lugar', con la finalidad específica de proponer una escala para su medición. El presupuesto fue el de que el atractivo de un lugar se evidencia, en gran parte, por la satisfacción que sienten sus habitantes, de modo que se hace necesario monitorear y emprender esfuerzos para elevar el nivel de satisfacción de este grupo de stakeholders. A partir de una revisión de la literatura del área, se definió el constructo y se evaluaron los principales indicadores de satisfacción. A continuación se emprendió un esfuerzo de construcción de una métrica, siguiendo los pasos convencionales de construcción de escalas para constructos con medición reflectiva. Para la validación empírica se recolectaron datos en algunas ciudades del estado de Paraíba, Brasil: João Pessoa, Campina Grande, Guarabira, y algunas otras pequeñas ciudades circundantes. Inicialmente se llevó a cabo una primera actividad de muestreo con la finalidad de limpiar la escala, y el segundo muestreo fue realizado para validar la misma. La escala final propuesta es unidimensional de cuatro ítems y puede ser utilizada no solo para ciudades, sino también para barrios y/o regiones. La propuesta de esa escala tiene una implicación práctica directa en la actuación de los gestores de lugares en el sentido de ofrecer un instrumento de medición de la satisfacción de sus habitantes. Desde el punto de vista académico, llena un vacío en la literatura del área en términos de métrica en el área de satisfacción de lugares.

Palabras clave: Residentes. Satisfacción. Medición. 


\section{INTRODUÇÃO}

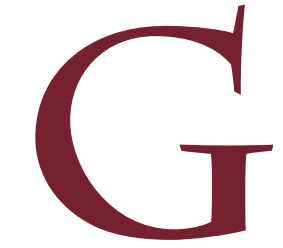
estores de lugares e os mais diversos agentes com interesse no sucesso de uma localidade (inclusive agentes do setor de turismo e de governo) normalmente operam com referências de espaços mais ou menos atrativos. Neste estudo, partimos do pressuposto de que entender os antecedentes e, consequentemente, elevar o nível de satisfação dos residentes é uma condição importante para incrementar a atratividade de um lugar, já que os residentes são um dos principais stakeholders dentro das atividades de planejamento turístico de cidades, regiões, países, dentre outros. Conforme argumentam Wang et al. (2010), a compreensão do que constitui a satisfação dos residentes possibilita um meio de alcançar o apoio ativo dos residentes no processo do desenvolvimento turístico de um lugar.

De acordo com Kotler, Haider e Rein (1993), para pensar em soluções de longo prazo, é importante que a comunidade se preocupe com os fatores básicos que a compõem (infraestrutura, atrações, imagem, qualidade de vida e pessoas), de forma a manter a satisfação dos cidadãos e atrair novos residentes, empresas, investidores e visitantes. A argumentação dos autores é bem justificada, pois, conforme relataremos no item 2 deste artigo, um elevado nível de satisfação dos residentes com seu lugar é um fator que atrai investimentos, compradores, turistas, visitantes, novos moradores, dentre outros. Isto gera, em consequência, valor econômico para o local, inclusive porque gera um referencial externo de qualidade de vida.

Defendemos o argumento de que os residentes são, na verdade, agentes ativos na configuração da atratividade de um lugar, uma vez que são parceiros e coprodutores das políticas, dos bens e dos serviços públicos (cf. ZENKER; PETERSON; AHOLT, 2013). Com efeito, os lugares dependem dos residentes para continuarem vivos e funcionando, seja econômica, social, cultural e/ou ambientalmente. Assim, a busca por manter a população de um lugar satisfeita deve ser o principal objetivo da gestão de lugares (INSCH; FLOREK, 2008; INSCH, 2010). Isto justifica, portanto, esforços de pesquisas para construção de métricas de satisfação e, ao mesmo tempo, para analisarmos empiricamente seus potenciais condicionantes e consequências. 
Dessa forma, a compreensão da satisfação dos residentes com o lugar é aperfeiçoada a partir de reflexões em nível conceitual e também em termos da medição do nível de satisfação, em um procedimento semelhante ao que fizemos para compreensão da satisfação de clientes com produtos e serviços de ofertantes diversos. Este artigo tem como objetivo contribuir com este desafio, a partir da proposição de uma escala de mensuração do nível de satisfação dos residentes com seus lugares.

O presente estudo está distribuído da seguinte maneira: após esse tópico introdutório, apresentaremos os principais recortes temáticos do estudo envolvendo a discussão conceitual de satisfação com o lugar e as suas variáveis de mensuração; na sequência, apresentamos o passo a passo da construção da escala; por fim, temos as considerações finais do estudo.

\section{REFERÊNCIAL TEÓRICO}

Levando em conta a finalidade do estudo, apresentamos aqui os principais componentes teóricos de referência e de fundamentos teóricos, em dois momentos: no item 2.1 apresentamos a discussão conceitual de satisfação dos residentes com o lugar e a relevância na área de marketing turístico e de lugares; e no item 2.2 apresentamos o resultado do levantamento sobre variáveis de mensuração, que fundamentaram o trabalho empírico.

\section{SATISFAÇÃO DOS RESIDENTES E RELEVÂNCIA PARA MARKETING TURÍSTICO E DE LUGARES}

A satisfação dos demandantes (sejam clientes de empresas ou cidadãos de um estado, por exemplo) vem sendo um dos temas mais recorrentes nas pesquisas acadêmicas e profissionais de marketing. Essa centralidade na teoria de marketing teve início ao final dos anos 50 do século passado, em torno do chamado marketing concept, momento em que o marketing deixou de ser visto como uma ferramenta para vender tudo que uma empresa ofertasse, e passou a defender uma preocupação com a satisfação de clientes e consumidores (KEITH, 1960, WEBSTER, 1988). 
Nesta perspectiva (que era associada a consumidores de ofertas de empresas), considerando que o consumidor precisava ser satisfeito, havia uma justificativa para que os mais diversos pensadores e pesquisadores de marketing empreendessem esforços no sentido de conceituar, medir e analisar os antecedentes e consequentes da satisfação do consumidor. A partir dos anos de 1970, quando houve a expansão do conceito de marketing e o entendimento de que o receptor do benefício pode ser qualquer agente a quem direcionamos um esforço de produção (e não necessariamente clientes de empresas), o conceito de satisfação precisou de uma expansão para além da visão restrita ao agente que adquire um produto (bem ou serviço de uma empresa). Este é o caso dos receptores -ou demandantes- no contexto de marketing social, de marketing turístico e de lugares, de marketing político, dentre outros, cuja oferta se distancia, conceitualmente, da ideia de bem ou serviço ofertado por uma empresa e consumidor por um cliente (BURGUETE, 2004).

Na conceituação convencional, a satisfação com um produto ou um serviço está associada à comparação entre o que os clientes esperam receber e o que efetivamente recebem, ou seja, é a comparação entre o desempenho percebido de um produto e as expectativas do cliente (GUSTAFSSON; JOHNSON; ROOS, 2005). Em uma perspectiva de marketing turístico e de lugares, esta definição ganha uma reconfiguração para se adaptar aos seus diversos agentes de interesse (os agentes são residentes, investidores, compradores, turistas...).

Para este artigo, tomamos um paralelo da definição convencional, e entenderemos que a satisfação com o lugar como a relação entre as expectativas dos agentes receptores do esforço de marketing turístico e de lugares em relação a determinados aspectos do lugar (gerais ou específicos), e como eles percebem o que efetivamente recebem do lugar em relação a esses aspectos. Do ponto de vista dos residentes, a definição é a mesma com foco específico neste agente.

Neste artigo partimos do entendimento de que, para que um lugar se torne atrativo para os demais stakeholders, em primeiro lugar os interesses da comunidade local devem ser atendidos e os residentes devem estar satisfeitos, inclusive porque, em princípio, são eles os principais interessados na ação dos gestores de um lugar. Para dar mais consistência a esse argumento, tomemos 
especificamente a perspectiva do turismo, que é a área de estudo mais desenvolvida quando pensamos no marketing de um lugar.

No início dos anos 1990, Kotler, Haider e Rein (1993) já afirmavam que um dos fatores de sucesso da ação de marketing é a satisfação dos cidadãos do lugar. Passadas mais de duas décadas desde esta afirmação, parece-nos que outros autores incorporaram esta ideia como pressuposto, a ponto de termos já em alguns estudos no campo de turismo uma clara preocupação em analisar o turismo com foco justamente no sujeito residente. Oviedo-Garcia, CastellanosVerdugo e Martin-Ruiz (2008), por exemplo, analisaram o apoio da comunidade local às atividades turísticas e detectaram que a promoção da participação dos residentes no planejamento turístico de uma localidade é essencial para a sustentabilidade e cooperação da comunidade local com o turismo. Já Mai, Rahtz e Shultz (2014) avaliaram a transição do desenvolvimento econônico no Vietnan e a contribuição oriunda da ativdade turística, enfatizando como critério de êxito de tal transição justamente o quanto ela alcançou benefício aos residentes.

Com efeito, não há sentido pensar em desenvolvimento turístico se isto não vier associado a um benefício maior para os sujeitos residentes, benefício este que alcance sua satisfação com lugar e a melhoria de sua qualidade de vida. Reafirmando o que já foi dito na introdução, uma orientação assim requer a construção de novas análises com a ênfase no residente, e isto passa pela disponibilidade de escalas de mensuração dos construtos associados ao residente. Em nível internacional, esta foi a motivação de Zenker, Petersen e Aholt (2013), que desenvolveram um índice de satisfação do residente. Aqui empreendemos um desafio semalhante, mas antes fazemos uma exposição do que já se pesquisou sobre o assunto.

\section{INDICADORES DE SATISFAÇÃO COM O LUGAR}

Nos estudos da área de satisfação com o lugar, a busca está em analisar fatores considerados essenciais para a geração dessa satisfação. Por exemplo, de acordo com Kotler, Haider e Rein (1993), os critérios que são considerados como geradores de satisfação em um lugar são: custo de vida, emprego, nível de criminalidade, oferta de estrutura para saúde e de agentes de saúde, 
transporte, educação, artes, lazer e clima. De forma mais resumida, Rainisto (2003) afirma que a atratividade de um lugar é oriunda da abertura de novas indústrias, muitas oportunidades de trabalho e nível de qualidade de vida.

Braun (2008) classifica os fatores geradores de satisfação de uma cidade de acordo com os stakeholders que, para o autor, são os residentes (buscam um lugar para viver), as empresas (buscam um lugar para fazer negócios), os visitantes (buscam um ambiente atrativo para visitar) e os investidores (buscam investir na cidade). Para o autor, os elementos importantes de um lugar para atender as necessidades e os desejos dos residentes seriam moradia e ambiente ao redor, acesso a emprego, acesso à educação, acesso ao lazer, acesso à família e aos amigos.

Já para as empresas seriam considerados os locais para se instalar e o ambiente ao redor, a oferta de colaboradores, os clientes e novos clientes, os fornecedores, as fontes de financiamento, por fim, o acesso a parceiros. No caso dos visitantes, são importantes os fatores de acomodação temporária e o ambiente ao redor, opções de atrações culturais e entretenimento, compras, locais de alimentação e oportunidades de negócios. Por fim, na visão de Braun (2008), para atender às necessidades e aos desejos dos investidores, são considerados os aspectos relativos ao mercado imobiliário e à atratividade de moradia, do ambiente de negócios e de visitação. Esses elementos estão resumidos conforme o Quadro 1.

Quadro 1 - Fatores de satisfação dos stakeholders de uma cidade

\begin{tabular}{|c|c|}
\hline STAKEHOLDER & FATORES DE SATISFAÇÃO COM O LUGAR \\
\hline Residentes & Moradia, emprego, educação, lazer, família e amigos. \\
\hline Empresas & $\begin{array}{l}\text { Lugar para se instalar, colaboradores, clientes, fornecedores, financiamento, } \\
\text { parcerias. }\end{array}$ \\
\hline Visitantes & $\begin{array}{l}\text { Acomodação, atrações culturais, compras, restaurantes, oportunidades de } \\
\text { negócios. }\end{array}$ \\
\hline Investidores & Mercado imobiliário, ambiente atrativo de moradia, de negócios e de visitaçã \\
\hline
\end{tabular}

Fonte: Braun (2008).

Insch e Florek (2008) afirmam que, especificamente no caso dos residentes, geralmente são avaliados aspectos de satisfação com o acesso à moradia, ao 
transporte, à saúde, à educação e à capacitação, ao lazer e locais de recreação, além de outras utilidades públicas, inclusive de oportunidades para interação social. Nesse mesmo sentido, Moser (2009) apresentou aspectos comuns considerados para promoção da satisfação com o ambiente em que o indivíduo vive, que seriam segurança, saúde e assistência social, ambiente doméstico, oportunidades para atividades de recreação e lazer, qualidade do ar, barulho, densidade do trânsito, clima, estrutura de transporte e oportunidades para adquirir novas informações e habilidades.

Em termos de fatores de satisfação com a vida na cidade, Insch (2010) elencou uma série de dimensões, que são: equilíbrio entre trabalho e vida pessoal (ex.: um bom emprego/carreira e tempo para lazer e para família); segurança pessoal e pública; o ambiente natural (ex.: paisagens, vistas, vida selvagem, etc.); os ambientes públicos da cidade (ex.: parque e jardins, prédios históricos, museus, universidades); cena cultural, artística e criativa (ex.: galerias, shows, festivais, teatro); abertura dos residentes para novas pessoas, novas ideias e diversidade; ambientes para a prática esportiva; localização relativa a outros destinos e acesso a outras cidades (ex.: conexões com outras cidades); e transporte público.

Em geral, esses indicadores servem de base para propostas de medição, o que se faz pela aferição de índices técnicos da dimensão objetiva de cada dimensão, ou pela avaliação da declaração do residente de seu nível de satisfação com cada dimensão, em uma perspectiva subjetiva. Análises dessa natureza são comumente baseadas em escalas de um único item, ou escalas de múltiplos itens, sendo possível fazer uma averiguação em perspectiva global ou com especificação por dimensão. Em qualquer dos contextos, é necessário que as escalas desenvolvidas sejam testadas e devidamente validadas.

Conforme indicado na introdução, neste artigo buscamos contribuir pela proposição de uma escala válida, e optamos por propor então uma métrica de múltiplos itens para mensuração do nível global de satisfação com o lugar. Para tanto, aplicamos um protocolo padrão de construção de escalas, com detalhamento no item seguinte. 


\section{CONSTRUÇÃO DA MEDIDA}

Na revisão da literatura de satisfação com o lugar percebemos a necessidade da construção de uma escala para medir a satisfação dos residentes com seus lugares de residência, em especial porque, em geral, os esforços neste sentido não foram suficientemente empreendidos. A construção dessas escalas se deu a partir do modelo proposto por Costa (2011), que definiu 10 passos de construção de medidas de construtos latentes. $O$ detalhamento está apresentado a seguir.

\section{- Passo 1: Especificação do domínio do construto}

Este passo consiste, fundamentalmente, na definição bem fundamentada do construto e de suas dimensões, se for o caso. Para esta pesquisa, o construto satisfação geral com o lugar foi apresentado, definido e suas dimensionalidades determinadas no item 2.1. Assim, o construto definido como indicado anteriormente foi tratado nesta pesquisa como unidimensional, por ser uma medida geral. Para efeito de medição, decidimos que o construto seria medido por itens com relação refletiva com o construto (ou seja, os itens selecionados para mensuração têm, por hipótese, sua variação definida pela variação do construto).

\section{-Passo 2: Geração de itens e validação de conteúdo}

Com a definição do construto e a determinação da natureza refletiva para os indicadores de medição, iniciamos a prospecção de itens. Nesta etapa, realizamos pesquisas na literatura especializada de marketing sobre satisfação do consumidor, inspirados em especial nos trabalhos de Gremler e Gwinner (2000), Kuo, Wu e Deng (2009), Jaislawe Niraj (2011), Siddiqui (2012) e Kaura (2013). A partir destes estudos, foram selecionados 15 itens nos critérios adotados (medirem satisfação em geral e terem sua variação oriunda da variação do nível de satisfação).

Estes 15 itens foram, em seguida debatidos, depurados e adaptados por nós à realidade brasileira e ao objeto de referência - a cidade de residência -, sendo ainda excluídos os itens repetitivos. Após estes procedimentos, excluímos 6 itens, 
restando um total de 9 que se mostraram, nesta primeira etapa, validados em termos de face e de conteúdo. Os itens finais estão dispostos no Quadro 2.

Quadro 2 - Itens propostos para a escala de satisfação geral com o lugar

\begin{tabular}{|c|c|}
\hline CÓDIGO & ITENS DA ESCALA \\
\hline S.GER.1 & O que tem na cidade onde resido é exatamente o que preciso \\
S.GER.2 & Comparando com outras cidades, eu estou muito satisfeito com a cidade onde habito \\
S.GER.3 & Eu estou satisfeito com o que tenho à disposição na cidade onde moro \\
S.GER.4 & Minha experiência com que tenho na cidade onde resido é satisfatória \\
S.GER.5 & Ser morador da cidade onde vivo tem sido uma experiência agradável para mim \\
S.GER.6 & Minha avaliação geral do que tenho à disposição na cidade onde resido é muito boa \\
S.GER.7 & Eu realmente gosto de morar na cidade onde vivo \\
S.GER.8 & De maneira geral eu estou satisfeito com a cidade onde resido \\
S.GER.9 & Eu acredito que a cidade onde vivo me fornece bons serviçOS \\
\hline
\end{tabular}

Fonte: Elaboração própria.

\section{-Passo 3: Decisões sobre as respostas}

Os formatos observados na literatura foram sempre de afirmações, modelo de enunciação que foi mantido nesta pesquisa (Quadro 2). Este tipo de enunciado tem a vantagem de permitir verificação da medida pela indicação da concordância com a afirmação, uma forma de verificação de boa familiaridade das pessoas em geral.

Adotamos como escala de verificação a de Likert em 10 pontos, variando de 1 (o nível de discordância total) até 10 (o nível de concordância total com a afirmação). A escala de 10 pontos tem a vantagem de ser uma referência bastante comum na população brasileira e facilita a indicação de resposta, além de permitir melhores operacionalizações estatísticas em possíveis aplicações da escala (COSTA, 2011). Pela lógica dos itens, graus de concordância mais elevados equivaleriam a níveis elevados de satisfação do residente com o seu lugar.

\section{- Passo 4: Construção do instrumento de pesquisa}

Para realização da primeira amostragem (passo 5 no modelo de Costa, 2011), os itens da escala foram organizados em um questionário, que foi impresso para aplicação direta (sem mediação por computador). As questões foram distribuídas de maneira aleatória, alternando-se entre questões de conteúdo 
socioeconômico e demográfico. O instrumento passou por um pré-teste e, em seguida, encaminhado para a primeira amostragem, detalhada no passo 5.

\section{- Passo 5: Primeira atividade de amostragem}

A primeira amostragem teve por finalidade gerar uma primeira rodada de resultados e viabilizar a realização de alguns testes exploratórios, com a finalidade estrita de limpeza da escala (procedimento realizado no passo 6). Para este passo, e visando dar heterogeneidade à amostra, decidimos buscar dados de respondentes residentes em duas cidades distintas do estado da Paraíba, no Nordeste do Brasil, a saber: João Pessoa (capital do estado, com população de 723.515 habitantes) e Guarabira (cidade de médio porte do interior do estado, com 55.326 habitantes) (IBGE, 2015).

Dada a finalidade exploratória desta amostragem, definimos que a coleta de dados seria feita em salas de aulas de universidades, faculdades, escolas técnicas, escolas de ensino médio, em locais de encontro de grupos religiosos e em outros aglomerados diversificados. A aplicação dos questionários deu-se no mês de maio de 2015 nos locais previamente determinados. A amostragem foi por conveniência e acessibilidade, e o trabalho de campo foi realizado pelos pesquisadores envolvidos na pesquisa, de modo que não houve necessidade de um treinamento prévio e acompanhamento para o trabalho externo.

Os dados coletados nessa primeira amostragem foram consolidados no software estatístico SPSS (versão 20) pelos próprios pesquisadores. Antes dos procedimentos de análise realizamos as análises preliminares de adequação dos dados, no sentido de identificar valores perdidos, erros e/ou valores discrepantes, sendo excluídos nove questionários com problemas. Obtivemos, após a retirada de valores perdidos, um total de 229 respondentes, sendo $148(64,6 \%)$ residentes na cidade de João Pessoa e 81 (35,4\%) residentes no município de Guarabira.

Observamos que, na amostra, 99 (43,2\%) dos respondentes afirmaram que nunca moraram em outra cidade além daquela em que residem, enquanto 130 (56,8\%) informaram que já residiram em outra cidade, o que denota um bom equilíbrio entre essas duas categorias. Concernente ao gênero, 
também percebemos uma boa divisão entre as categorias, com 120 (52,4\%) pertencentes ao sexo masculino, e 109 (47,6\%) ao sexo feminino. A maioria das pessoas da amostra estava cursando ou havia concluído o ensino superior ou pós-graduação, totalizando em 146 (63,8\%), ao passo que 81 (35,4\%) dos respondentes tinha somente o ensino médio, e 2 (0,9\%) respondentes tinham somente o ensino fundamental. Esses resultados sinalizam adequação da amostra em termos de sua heterogeneidade, o que assegura condições adequadas para o procedimento seguinte.

- Passo 6: Procedimentos de limpeza da escala

Conforme já sinalizado, o procedimento de limpeza dos dados consiste em analisar o comportamento dos itens de mensuração e excluir aqueles que não apresentam adequação às propriedades psicométricas de correlação bivariada, de consistência interna e de adequação fatorial.

Nestes termos, realizamos inicialmente a extração da matriz de correlações (Pearson), cujos resultados estão indicados na Tabela 1. De posse dos resultados, pudemos observar que nenhum par de variáveis teve correlação estatisticamente nula (todos significativos a $\mathrm{p}<0,001$ ) e que todos os pares obtiveram correlações moderadas (entre 0,40 e 0,80). Levando em conta que a suposição dos itens é de que sejam refletivos em relação ao construto latente, entendemos que, por esta análise preliminar, todos os itens estão adequados em termos de medição do construto.

Tabela 1 - Matriz de correlação da satisfação geral com a cidade

\begin{tabular}{lcccccccc}
\hline & S.GER.1 & S.GER.2 & S.GER.3 & S.GER.4 & S.GER.5 & S.GER.6 & S.GER.7 & S.GER.8 \\
\hline S.GER.2 &, 584 & & & & & & & \\
S.GER.3 &, 733 &, 669 & & & & & & \\
S.GER.4 &, 575 &, 614 &, 632 & & & & & \\
S.GER.5 &, 499 &, 681 &, 588 &, 716 & & & & \\
S.GER.6 &, 583 &, 672 &, 675 &, 665 &, 657 & & & \\
S.GER.7 &, 456 &, 683 &, 552 &, 554 &, 700 &, 666 & & \\
S.GER.8 &, 560 &, 753 &, 655 &, 648 &, 683 &, 730 &, 796 & \\
S.GER.9 &, 631 &, 628 &, 649 &, 567 &, 553 &, 608 &, 519 &, 667 \\
\hline
\end{tabular}

Fonte: Dados da pesquisa (2015).

No procedimento de verificação da consistência interna (que é uma indicadora de confiabilidade da escala), obtivemos um alpha de Cronbach de 0,939, o que 
demonstra um nível muito bom de confiabilidade. Na sequência, verificamos que, nas saídas do SPSS, não obteríamos um nível melhor do alpha a partir da exclusão de algum item (este nível do alpha tem influência, potencialmente, do número de variáveis - 9 ao total -, uma vez que o alpha tende a crescer com o tamanho da amostra de itens; cf. Costa, 2011). Assim, a sinalização é de que os itens mensuram de forma confiável o construto latente, e não há indicação de necessidade de exclusão de algum item.

Posteriormente, verificamos a adequação da amostra de itens para a análise fatorial e obtivemos uma medida de teste KMO de 0,926, com estatística de Barlett com $\chi^{2}=1592,639$ (36 graus de liberdade), significativo a $p<0,001$. Estes valores indicam a adequação da amostra de itens para a realização da análise fatorial. Na extração dos fatores, pudemos observar a existência de apenas um autovalor acima de 1, com um nível de explicação da variância total considerado satisfatório (67,53\%). Tal resultado nos sugeriu que o conjunto de variáveis possui apenas um fator subjacente, conforme esperado.

Dado o intuito de validar a escala, o método de extração utilizado foi o de máxima verossimilhança em substituição do método de componentes principais (procedimento padrão em análise exploratória), tendo em vista que o primeiro (máxima verossimilhança) é um método confirmatório e gera escores reduzidos comparativamente ao segundo ${ }^{1}$, sendo, portanto, mais rigoroso na indicação do nível de associação dos itens ao construto subjacente.

Na Tabela 2 apresentamos os resultados dos escores fatoriais das variáveis e das comunalidades, sendo possível observar que todos os itens apresentaram escores elevados (acima de 0,7 ), sinalizando boa adequação na estrutura fatorial. A partir desses resultados, percebemos que, também na análise fatorial, não há a sugestão de retirada de nenhum item.

1 O teste de hipótese da análise fatorial confirmatória (que testa a igualdade da matriz de correlação estimada pelos escores fatoriais com a matriz de correlação observada) é sensível ao tamanho da amostra, de modo que, mesmo com uma estrutura fatorial adequada, é possível que o teste não seja significativo ao nível de significância adequado (aqui de $5 \%$ ). Isto sinaliza que os resultados poderão ser considerados como adequados independente do teste de hipóteses da extração. 
Tabela 2 - Escores e comunalidades do construto satisfação geral com a cidade $-1^{\text {a }}$ extração

\begin{tabular}{|c|c|c|c|}
\hline Código & Itens & Esc. & Com. \\
\hline SAT.GER.1 & O que tem na cidade onde resido é exatamente o que preciso & ,703 &, 495 \\
\hline SAT.GER.2 & $\begin{array}{l}\text { Comparando com outras cidades, eu estou muito satisfeito com a cidade } \\
\text { onde habito }\end{array}$ &, 838 &, 702 \\
\hline SAT.GER.3 & Eu estou satisfeito com o que tenho à disposição na cidade onde moro & ,793 &, 628 \\
\hline SAT.GER.4 & Minha experiência com que tenho na cidade onde resido é satisfatória & ,773 &, 597 \\
\hline SAT.GER.5 & $\begin{array}{l}\text { Ser morador da cidade onde vivo tem sido uma experiência agradável para } \\
\text { mim }\end{array}$ &, 800 &, 640 \\
\hline SAT.GER.6 & $\begin{array}{l}\text { Minha avaliação geral do que tenho à disposição na cidade onde resido é } \\
\text { muito boa }\end{array}$ &, 829 &, 688 \\
\hline SAT.GER.7 & Eu realmente gosto de morar na cidade onde vivo &, 797 &, 635 \\
\hline SAT.GER.8 & De maneira geral eu estou satisfeito com a cidade onde resido &, 879 & ,773 \\
\hline SAT.GER.9 & Eu acredito que a cidade onde vivo me fornece bons serviços &, 747 &, 558 \\
\hline
\end{tabular}

Fonte: Dados da pesquisa (2015).

No conjunto das três verificações (correlação, alpha e análise fatorial), os resultados sinalizam que, ao considerarmos as medidas, o conjunto de itens mensura adequadamente o construto 'satisfação com a cidade'. Porém, antes de dar o resultado por consolidado e considerando que esta amostragem teve por finalidade proceder à limpeza desta escala, optamos por aprofundar a análise do conjunto de itens levando em conta o resultado em conjunto com o conteúdo dos itens.

Na releitura atenta dos conteúdos, observamos um potencial problema no fato de dois deles destoarem dos demais, por refletirem avaliações globais, diferentemente dos demais itens (SAT.GER.6 - "Minha avaliação geral do que tenho à disposição na cidade onde resido é muito boa"; e SAT.GER.8 "De maneira geral eu estou satisfeito com a cidade onde resido"). Conforme Rossiter (2011), este tipo de item promove um viés de mensuração em termos de conteúdo, além de inflacionar as medidas das extrações fatoriais e de consistência interna.

Por este entendimento, os itens foram excluídos e mais uma iteração da análise fatorial foi procedida. Como resultados do teste de ajustamento, obtivemos um valor de $\mathrm{KMO}$ de 0,898, e os resultados do teste de Barlett com $\chi^{2}=100,877$, 
$g l=4$ e $p<0,001$. Tais resultados nos sinalizam uma ótima adequação dos itens remanescentes para seguirmos para a realização da análise fatorial.

Os resultados dos autovalores indicaram que apenas um fator emergiu com autovalor maior que 1 , e este fator explicando $66,54 \%$ da variância total. 0 resultado do alpha de Cronbach foi de 0,915. Na Tabela 3 apresentamos os escores fatoriais e as comunalidades dessa segunda extração, sendo possível observar escores fatoriais elevados após a exclusão dos dois itens.

Tabela 3 - Escores e comunalidades do construto satisfação geral com a cidade $-2^{a}$ extração

\begin{tabular}{|c|c|c|c|}
\hline Código & Itens & Escore & Com. \\
\hline SAT.GER.1 & O que tem na cidade onde resido é exatamente o que preciso & ,739 &, 546 \\
\hline SAT.GER.2 & $\begin{array}{l}\text { Comparando com outras cidades, eu estou muito satisfeito com a cidade } \\
\text { onde habito }\end{array}$ & 831 & ,691 \\
\hline SAT.GER.3 & Eu estou satisfeito com o que tenho à disposição na cidade onde moro & 816 & ,666 \\
\hline SAT.GER.4 & Minha experiência com que tenho na cidade onde resido é satisfatória & ,782 & ,612 \\
\hline SAT.GER.5 & $\begin{array}{l}\text { Ser morador da cidade onde vivo tem sido uma experiência agradável } \\
\text { para mim }\end{array}$ & 801 & ,642 \\
\hline SAT.GER.7 & Eu realmente gosto de morar na cidade onde vivo & ,743 &, 553 \\
\hline SAT.GER.9 & Eu acredito que a cidade onde vivo me fornece bons serviços & 752 & ,565 \\
\hline
\end{tabular}

Fonte: Dados da pesquisa (2015).

Consideramos que esta segunda extração assegura que a escala final mantém uma sólida estrutura psicométrica, além de estar mais adequada do ponto de vista de validade de conteúdo e de face. A escala final desse construto ficou, portanto, com sete itens, e adequada para análise subsequente, com as amostragens adicionais.

- Passo 7: Trabalhos de campo adicionais

Os trabalhos de campo adicionais são orientados a testar novamente a escala e a proceder a novas limpezas, caso seja necessário. Preferencialmente, a amostra deve ser de respondentes que serão efetivamente aqueles de interesse na aplicação da escala (COSTA, 2011). A partir dos itens remanescentes oriundos do trabalho de limpeza da primeira amostragem, decidimos acrescentar novas questões no questionário. O ordenamento e as questões demográficas foram 
mantidos e foram inseridos itens de mensuração de satisfação com fatores específicos da cidade, com a medição com um único item por dimensão, conforme Quadro 3. A escala de verificação destes itens foi de 10 pontos, em que 1 indicada 'baixo nível', e 10 indicava 'alto nível', com os demais pontos indicando níveis intermediários de satisfação entre estes dois extremos.

Quadro 3 - Fatores específicos de satisfação com a cidade

\begin{tabular}{|c|l|}
\hline DIMENSÃO & ITEM \\
\hline CONSUMO & $\begin{array}{l}\text { Entendo que as condições de acesso ao consumo na cidade onde moro são } \\
\text { satisfatórias em }\end{array}$ \\
\hline INFRAESTRUTURA & $\begin{array}{l}\text { Para mim, as condições de lazer na cidade onde habito são satisfatórias em } \\
\text { satisfatórias em }\end{array}$ \\
\hline EDUCAÇÃO & $\begin{array}{l}\text { Eu considero que as condições de educação na cidade onde vivo são satisfatórias } \\
\text { em }\end{array}$ \\
\hline $\begin{array}{c}\text { EXERCÍCIO } \\
\text { PROFISSIONAL }\end{array}$ & $\begin{array}{l}\text { Eu considero que as condições de exercício profissional na cidade onde habito } \\
\text { são satisfatórias em }\end{array}$ \\
\hline SAÚDE & Para mim, as condições de saúde na cidade onde moro são satisfatórias em \\
\hline SEGURANÇA & Para mim, as condições de segurança na cidade onde vivo são satisfatórias em \\
\hline INTERAÇÃO SOCIAL & $\begin{array}{l}\text { Eu acho que as oportunidades para interação social na cidade onde vivo são } \\
\text { satisfatórias em }\end{array}$ \\
\hline
\end{tabular}

Fonte: Elaboração própria.

Devido a limitações de tempo e de recursos, decidimos realizar somente mais uma amostragem. Assim, neste segundo esforço de coleta buscamos coletar dados, além das cidades de João Pessoa e Guarabira, na cidade de Campina Grande, também localizada no estado da Paraíba (com população de 385.276 habitantes) (IBGE, 2015).

A coleta dos dados foi realizada no mês de junho de 2015 e buscamos obter uma amostra mais diversificada de respondentes, aplicando questionários em rodoviárias, igrejas, aeroportos, universidades, faculdades e escolas. O esforço de coleta foi conduzido pelos próprios pesquisadores, não havendo a necessidade de treinamento ou acompanhamento do trabalho de campo. Após a retirada de 16 questionários inválidos por problema de resposta incompleta e outliers, tivemos uma amostra final com 415 respondentes. Destes, 139 eram residentes em João Pessoa, 114 em Campina Grande, 53 em Guarabira e 109 de outras cidades circunvizinhas. 
Em termos de situação laboral dos respondentes, verificamos que $55,4 \%$ não estavam trabalhando e 44,6\% exerciam alguma atividade profissional (seja em meio turno ou o dia inteiro). A maioria dos respondentes era jovem, com 44,6\% tendo até 20 anos de idade e 33,7\% tendo entre 21 e 30 anos. Encontramos uma situação equilibrada entre os gêneros, sendo $46,5 \%$ do sexo masculino e 53,5 do sexo feminino.

A renda familiar dos respondentes se concentrou em 55,2\% em até $R \$$ $2.000,00$ mensais e $26,5 \%$ com renda familiar entre $R \$ 2.000,00$ e $R \$ 4.000,00$ mensais. Referente ao grau de escolaridade dos residentes, obtivemos resultados equilibrados entre ensino médio (41\%) e ensino superior (46,6\%). Além disso, $53 \%$ dos respondentes afirmaram que já moraram em outra cidade, contra $47 \%$ que afirmaram nunca haver morado em outra cidade além da que residem atualmente. Por fim, observamos uma média de aproximadamente 19 anos de tempo de moradia na cidade em que o respondente morava atualmente, o que denota um considerável tempo de experiência com a cidade de residência, tendo assim uma boa base para emitir opiniões. De maneira geral, podemos dizer que a amostra tem características heterogêneas adequadas para as análises cabíveis nesse segundo processo de amostragem.

- Passo 8: Procedimentos adicionais de limpeza da escala

Como procedimento adicional de limpeza da escala, procedemos à análise fatorial confirmatória, por meio do software AMOS (versão 15), que permite a extração fatorial com um conjunto adicional de medidas de ajustamento, além dos testes de hipóteses sobre os escores fatoriais. Foram procedidas diversas iterações, na busca de encontrar resultados estatísticos que compatibilizassem, ao final, uma boa adequação em termos de medida com um bom ajuste de conteúdo de cada item. Após as iterações, e adotando como critério de exclusão baixos escores fatoriais ou a melhoria agregada nas medidas de ajustamento, foram excluídas três variáveis. A escala final ficou, portanto, com quatro itens. 
Tabela 4 - Análise fatorial confirmatória da satisfação geral com a cidade na $2^{\mathrm{a}}$ amostragem

\begin{tabular}{|c|c|c|c|}
\hline \multicolumn{2}{|c|}{ Variáveis } & Escores & $\begin{array}{c}\text { Valores t } \\
(\mathrm{CR})^{*}\end{array}$ \\
\hline \multicolumn{4}{|c|}{ PAINEL 1 - ITENS E ESCORES FATORIAIS } \\
\hline \multicolumn{2}{|c|}{ O que tem na cidade onde resido é exatamente o que preciso } & 0,778 & 17,407 \\
\hline \multicolumn{2}{|c|}{ Eu estou satisfeito com o que tenho à disposição na cidade onde moro } & 0,880 & 20,187 \\
\hline \multicolumn{2}{|c|}{ Minha experiência com que tenho na cidade onde resido é satisfatória } & 0,816 & 18,510 \\
\hline \multicolumn{2}{|c|}{ Eu acredito que a cidade onde vivo me fornece bons serviços } & 0,815 & - \\
\hline \multicolumn{4}{|c|}{ PAINEL 2 - MEDIDAS DE AJUSTAMENTO } \\
\hline Medida de ajustamento & Observado & \multicolumn{2}{|c|}{$\begin{array}{l}\text { Requisito de } \\
\text { adequação }\end{array}$} \\
\hline Qui-quadrado $\left(\chi^{2}\right)$ & 6,972 & \multicolumn{2}{|r|}{ - } \\
\hline Graus de liberdade (gl) & 2 & \multicolumn{2}{|r|}{-} \\
\hline Razão $\chi^{2} / g l$ & 3,486 & \multicolumn{2}{|c|}{$<5,000$} \\
\hline p-valor & 0,031 & \multicolumn{2}{|c|}{$>0,050$} \\
\hline GFI & 0,991 & \multicolumn{2}{|c|}{$>0.900$} \\
\hline CFI & 0,995 & \multicolumn{2}{|c|}{$>0.900$} \\
\hline RMSEA & 0,077 & \multicolumn{2}{|c|}{$<0,080$} \\
\hline
\end{tabular}

* Todos os valores significativos a $p<0,001$

Fonte: Dados da pesquisa (2015).

Os resultados que estão expostos na Tabela 4 mostram uma adequação muito boa dos escores (todos acima de 0,77 e estatisticamente não nulos). As medidas de ajustamento estão todas dentro dos limites sugeridos na literatura de mensuração, com exceção do p-valor do teste de qui-quadrado (este resultado é comum quando trabalhos com amostras razoavelmente grandes, como a desta pesquisa, o que motiva a utilização das demais medidas (cf. COSTA, 2011)). Nestes termos, entendemos que os resultados sinalizam uma boa adequação da amostra de itens para mensuração do construto latente.

- Passo 9: Análise de validade e de confiabilidade da escala final

Esta etapa tem por finalidade apresentar evidências adicionais de validade. Convencionalmente, são apresentadas as validades convergente e discriminante. Na primeira (validade convergente, que sinaliza o conjunto de itens adequado em termos de medidas repetidas do construto), e adotando os referenciais da literatura especializada, tomamos os valores de critical ratio (CR) da saída do 
AMOS como indicadores de adequação de validade. Observamos que todos os valores foram estatisticamente não nulos (a $p<0,05)$, o que sinaliza que os itens têm sua variação efetivamente oriunda do construto latente. Em termos de validade discriminante (que sinaliza o quanto a medida difere de outras das quais deve diferir), aqui não tivemos como proceder a avaliações devido ao fato de o construto ter medido de forma unidimensional, em conformidade com os objetivos da pesquisa.

Por fim, como sinalização de validade de critério, verificamos se uma medida geral da escala varia de forma concomitante com as medições de dimensões específicas do lugar. Foi adotada como medida de sinalização a correlação (Pearson) bivariada da escala agregada (pela média dos escores de cada respondente) com os itens das dimensões de satisfação (ver Quadro 3), e entendemos que havia indicação de validade se houvesse correlação não nula e de nível pelo menos moderado. Os resultados são apresentados na Tabela 5, e a sinalização é de que, também por este critério, os itens possuem validade na medição do construto latente (as correlações foram pelo menos em nível moderadas e não nulas estatisticamente, a $p<0,001$ ).

Tabela 5 - Correlação satisfação geral com específicos

\begin{tabular}{c|c}
\hline Dimensões & Satisfação geral \\
\hline Condições de acesso ao consumo & 0,627 \\
Condições de educação & 0,620 \\
Condições de infraestrutura & 0,607 \\
Condições de lazer & 0,636 \\
Condições de exercício profissional & 0,614 \\
Condições de saúde & 0,536 \\
Condições de segurança & 0,477 \\
Oportunidades para interação social & 0,550 \\
\hline
\end{tabular}

Fonte: Dados da pesquisa (2015).

Por fim, a análise de confiabilidade foi feita pela análise de consistência interna, por meio do coeficiente alpha de Cronbach. O valor final dos quatro itens foi de 0,893, o que indica elevada consistência interna no conjunto de itens, não havendo melhoria com a potencial exclusão de algum destes. 
- Passo 10: Desenvolvimento de normas e recomendações

A última etapa consiste na recomendação de aplicação da escala. Entendemos que a escala desenvolvida, embora tenha sido testada em cidades, pode ser utilizada também em bairros, estados ou regiões, e os resultados podem permitir a realização de comparações diversas.

Dentro do instrumento de pesquisa, a recomendação é de que os itens sejam distribuídos de maneira aleatória e separados por outras variáveis como forma de evitar vieses nas respostas. Também não é recomendado que esse construto seja analisado com temas pouco associados, pois pode gerar indisposição do respondente.

Como referência de aplicação da escala, o número de pontos fica a critério do pesquisador, levando em conta a capacidade do respondente, porém as pesquisas têm mostrados que escalas com 10 pontos geram sempre bons resultados. Como indicação da forma de agregação dos itens, recomendamos que seja extraída a média dos escores por respondente, pois isto permite a extração de medidas na mesma escala de aplicação (1 a 5, 1 a 10...). Em qualquer opção, e considerando o enunciado dos itens, a interpretação será baseada no nível médio do escore agregado (por respondentes), de tal modo que escores (e, portanto, médias) mais próximos do extremo superior indicarão níveis elevados de satisfação com o lugar, e escores mais próximos do limite inferior indicarão níveis baixos.

\section{CONSIDERAÇÕES FINAIS}

Conforme proposta apresentada no início deste estudo, o nosso objetivo foi desenvolver uma escala de mensuração da satisfação geral dos residentes com seus lugares. Neste sentido, podemos afirmar que o objetivo da pesquisa foi satisfatoriamente alcançado, com a geração de uma métrica baseada em quatro afirmações para averiguação por meio de uma escala de verificação do tipo Likert. A escala final, gerada a partir do conjunto de refinamentos empreendidos, é apresentada no Quadro 4. 
Quadro 4 - Escala proposta de satisfação com o lugar

\begin{tabular}{|c|}
\hline ITENS \\
\hline O que tem na cidade onde resido é exatamente o que preciso \\
Minha experiência com que tenho na cidade onde resido é satisfatória \\
Eu acredito que a cidade onde vivo me fornece bons serviços
\end{tabular}

Fonte: Elaboração própria.

Os resultados desta pesquisa possuem implicação prática no favorecimento da atuação dos gestores de lugares, que podem usar a escala para aferições do nível de satisfação dos residentes com o lugar em que vivem, além de buscarem promover (ou manter) ações que possam elevar (ou manter) o nível de satisfação verificado. Conforme já argumentado, um lugar cujos residentes têm uma boa avaliação do que a(o) cidade/bairro/região oferece, é um lugar atrativo para investimentos, novasempresas, novosresidentes, visitanteseturistas, oquefomenta o desenvolvimento econômico do lugar. Deste modo, o monitoramento do nível de satisfação do residente é uma demanda que se apresenta periodicamente aos gestores, o que requer um instrumento rigorosamente validado para esta verificação, como é o caso da escala aqui proposta.

Em termos acadêmicos, a própria criação de escala de satisfação geral com o lugar já constitui em si uma contribuição relevante, visto que não havia (ou ao menos não identificamos) na literatura de turismo e gestão pública uma escala que medisse, adequadamente, esse construto. Nesse sentido, essa pesquisa traz uma contribuição para os esforços de mensuração do construto satisfação com o lugar, para potenciais operacionalizações deste construto em outros estudos, inclusive envolvendo outros construtos (como qualidade de vida, por exemplo).

Algumas limitações foram identificadas no decorrer deste estudo. Em especial, destacamos o fato de a escala proposta ser para operacionalização unidimensional do construto. Embora isto não seja um problema maior, recomendamos que sejam empreendidos esforços acadêmicos de dimensionamento e construção de uma métrica de múltiplos itens para as diferentes dimensões. Além disto, entendemos haver uma limitação em termos da amostra, que foi restrita a três cidades. Sugerimos, portanto, a aplicação da escala e a sua validação em outras cidades. 
BRAUN, E. City Marketing: Towards an integrated approach. Erasmus University Rotterdam, 2008.

BURGUETE, J. L. V. Pasado, presente y futuro de las dimensiones pública y social en el desarrollo conceptual del marketing. International Review on Public and Nonprofit Marketing, v. 1, n.1, p. 9-34, 2004.

COSTA, F. J. Mensuração e desenvolvimento de escalas: aplicações em administração. Rio de Janeiro: Editora Ciência Moderna, 2011.

FERNANDES, L. M. M.; CORIOLANO, L. M. N. T. A governança na política nacional de regionalização do turismo: estudo nos grupos gestores dos destinos indutores do Ceará. Revista Turismo - Visão e Ação - Eletrônica, v. 17, n. 2, 2015.

GREMLER, D. D.; GWINNER, K. P. Customer-employee rapport in service relationships. Journal of Service Research, v.3, n. 1, p. 82-104, 2000.

GUSTAFSSON, A.; JOHNSON, M. D. ; ROOS, I. The effects of customer satisfaction, relationship commitment dimensions, and triggers on customer retention. Journal of marketing, $p$. 210-218, 2005.

IBGE - Instituto Brasileiro de Geografia e Estatística. Cidades. Disponível em: <http://www. ibge.gov.br/cidadesat/link.php?uf=pb>. Acesso em 29 mar 2015.

INSCH, A. ; FLOREK, M. A great place to live, work and play: conceptualising place satisfaction in the case of a city's residents. Journal of Place Management and Development, v.1, $n$. 2, p. 138-149, 2008.

INSCH, A. Managing residents' satisfaction with city life: Application of ImportanceSatisfaction analysis. Journal of Town and City Management, v. 1, n. 2, p. 164-174, 2010.

JAISWAL, A. K.; NIRAJ, R. Examining mediating role of attitudinal loyalty and nonlinear effects in satisfaction-behavioral intentions relationship. Journal of Services Marketing, v. 25, n. 3, 165-175, 2011.

KAURA, V. Antecedents of customer satisfaction: a study of public and private sector banks. International Journal of Bank Marketing, v. 31, n. 3, p. 3-33, 2013.

KEITH, R. J. The marketing revolution. The Journal of Marketing, p. 35-38, 1960.

KOTLER, P.; HAIDER, D. H.; REIN, I. Marketing Places: attracting investment, industry, and 
tourism to cities, states, and nations. New York: MaxwellMacmillan International, 1993.

KUO, Y. F.; WU, C. M.; DENG, W. J. The relationships among service quality, perceived value, customer satisfaction, and post-purchase intention in mobile value-added services. Computers in Human Behavior, v. 25, n. 4, p. 887-896, 2009.

MAI, N. T. T.; RAHTZ, D. R.; SHULTZ, C. J. Tourism as Catalyst for Quality of Life in Transitioning Subsistence Marketplaces:Perspectives from Ha Long, Vietnam. Journal of Macromarketing, v. 34, n. 1, p. 28-44, 2014.

MOSER, G. Quality of life and sustainability: Toward person-environment congruity. Journal of Environmental Psychology, v. 29, n. 3, p. 351-357, 2009.

OVIEDO-GARCIA, M. A.; CASTELLANOS-VERDUGO, M.; MARTIN-RUIZ, D. Gaining residents' support for tourism and planning. International Journal of Tourism Research, v. 10, p. 95-109, 2008.

RAINISTO, S. K. Success factors of place marketing: A study of place marketing practices in Northern Europe and the United States. Helsinki University of Technology, 2003.

ROSSITER, J. R. Measurement for the social sciences: The C-OAR-SE method and why it must replace psychometrics. Springer, 2011.

SIDDIQUI, K. Personality influences on customer satisfaction. African Journal of Business Management, v. 6, n. 11, p. 4134-4141, 2012.

WANG, X.; ZHEN, F.; WU, X. G.; ZHANG, H.; LIU, Z. H. Driving factors of resident satisfaction with tourism development: A case study of Yangshuo in Guangxi Zhuang Autonomous Region [J]. Geographical Research, v. 5, 2010.

WEBSTER, F. E. The rediscovery of the marketing concept. Business horizons, v. 31, n. 3, p. 29-39, 1988.

ZENKER, S.; PETERSEN, S.; AHOLT, A. The Citizen Satisfaction Index (CSI): Evidence for a four basic factor model in a German sample. Cities, v. 31, p. 156-164, 2013. 